\title{
Higher-Order Approximate Periodic Solutions of a Nonlinear Oscillator with Discontinuity by Variational Approach
}

\author{
M. Orhan Kaya, ${ }^{1}$ S. Altay Demirbağ, ${ }^{2}$ and F. Özen Zengin ${ }^{2}$ \\ ${ }^{1}$ Faculty of Aeronautics and Astronautics, Istanbul Technical University, Maslak 34469, Istanbul, Turkey \\ ${ }^{2}$ Faculty of Science and Letters, Istanbul Technical University, Maslak 34469, Istanbul, Turkey \\ Correspondence should be addressed to M. Orhan Kaya, kayam@itu.edu.tr
}

Received 16 October 2008; Revised 12 March 2009; Accepted 14 May 2009

Recommended by Ekaterina Pavlovskaia

He's variational approach is modified for nonlinear oscillator with discontinuity for which the elastic force term is proportional to $\operatorname{sgn}(\mathrm{u})$. Three levels of approximation have been used. We obtained $1.6 \%$ relative error for the first approximate period, $0.3 \%$ relative error for the secondorder approximate period. The third approximate solution has the accuracy as high as $0.1 \%$.

Copyright (C) 2009 M. Orhan Kaya et al. This is an open access article distributed under the Creative Commons Attribution License, which permits unrestricted use, distribution, and reproduction in any medium, provided the original work is properly cited.

\section{Introduction}

Considerable attention has been directed toward the solution of nonlinear equations since they play crucial role in applied mathematics, physics, and engineering problems. In general, the analytical approximation to solution of a given nonlinear problem ismore difficult than the numerical solution approximation. During the past decades, several types of methods are proposed to obtain approximate solution of nonlinear equations of various types. Among them are variational iteration methods [1-7], homotopy perturbation method [815], modified Lindstedt-Poincare method [16], parameter expansion method [17, 18], and variational methods [19-21]. The variational method is different from any other variational methods in open literature, and it is only valid for nonlinear oscillators [22]. Paper [23] is an example of use of variational approach method in nonlinear oscillator problem.

When we examine the frequency amplitude relations of some nonlinear oscillators, it is seen that paper [24] focuses on only the first-order solutions.

Variational methods combine the following two advantages: (1) they provide physical insight into the nature of the solution of the problem; (2) the obtained solutions are the best among all the possible trial functions [20]. 
In the present study, we have investigated the application of variational approach to nonlinear oscillator with discontinuity.

\section{A Variational Method}

Let us consider a general nonlinear oscillator in the form

$$
u^{\prime \prime}+f(u)=0
$$

He proposed a variational principle for (2.1) as follows [20]:

$$
J(u)=\int_{0}^{T / 4}\left(-\frac{1}{2} u^{\prime 2}+F(u)\right) d t
$$

where $T$ is period of the nonlinear oscillator, $\partial F / \partial u=f$. Actually, the upper limit is originally $T$ instead of $T / 4$. Normally, it works in most of the cases. Let us suppose that $f(u)=\operatorname{sgn}(u)$ such

$$
\operatorname{sgn}(u)= \begin{cases}-1, & u<0 \\ +1, & u \geq 0\end{cases}
$$

therefore

$$
F(u)= \begin{cases}-u, & u<0, \\ u, & u \geq 0 .\end{cases}
$$

But this form is not suitable for discontinuity equation. Therefore, we propose the equation in the form of

$$
J(u)=\int_{0}^{T / 2}\left(-\frac{1}{2} u^{\prime 2}+|u|\right) d t
$$

Assume that its solution can be expressed as

$$
u(t)=A \cos \omega t+B(\cos \omega t-\cos 3 \omega t)+C(\cos 3 \omega t-\cos 5 \omega t)+\cdots,
$$

where $\omega$ is the frequency of the oscillator. 
Inserting (2.6) into (2.5) yields

$$
\begin{aligned}
& J(A, B, C, \ldots, \omega) \\
& =\int_{0}^{T / 2}\left\{-\frac{1}{2} \omega^{2}[A \sin \omega t+B(\sin \omega t-3 \sin 3 \omega t)+C(3 \sin 3 \omega t-5 \sin 5 \omega t)+\cdots]^{2}\right. \\
& \quad+|A \cos \omega t+B(\cos \omega t-\cos 3 \omega t)+C(\cos 3 \omega t-\cos 5 \omega t)+\cdots|\}
\end{aligned}
$$

Let us define $\tau=\omega t$. Then (2.7) becomes

$$
\begin{aligned}
J(A, B, C, . . . \omega)=\int_{0}^{\pi}\{ & -\frac{1}{2} \omega[A \sin \tau+B(\sin \tau-3 \sin 3 \tau)+C(3 \sin 3 \tau-5 \sin 5 \tau)+\cdots]^{2} \\
& \left.+\frac{1}{\omega}|A \cos \tau+B(\cos \tau-\cos 3 \tau)+C(\cos 3 \tau-\cos 5 \tau)+\cdots|\right\} d \tau .
\end{aligned}
$$

Using the Ritz method, we require

$$
\begin{gathered}
\frac{\partial J}{\partial \omega}=0, \quad \frac{\partial J}{\partial A}=0, \quad \frac{\partial J}{\partial B}=0, \quad \frac{\partial J}{\partial C}=0, \ldots, \\
\frac{\partial J}{\partial \omega}=-\int_{0}^{\pi}\left\{\frac{1}{2}[A \sin \tau+B(\sin \tau-3 \sin 3 \tau)+C(3 \sin 3 \tau-5 \sin 5 \tau)+\cdots]^{2}\right. \\
\left.+\frac{1}{\omega^{2}}|A \cos \tau+B(\cos \tau-\cos 3 \tau)+C(\cos 3 \tau-\cos 5 \tau)+\cdots|\right\} d \tau .
\end{gathered}
$$

By a careful inspectation, we find that

$$
\frac{\partial J}{\partial \omega}<0
$$

Thus, the conditions in (2.9) reduce to

$$
\frac{\partial J}{\partial A}=0, \quad \frac{\partial J}{\partial B}=0, \quad \frac{\partial J}{\partial C}=0, \ldots
$$

\section{Application}

Consider the following nonlinear oscillator with discontinuity:

$$
u^{\prime \prime}+\operatorname{sgn}(u)=0
$$


with initial conditions

$$
u(0)=A, \quad \frac{d u(0)}{d t}=0
$$

Its variational formulation can be written as follows:

$$
J(u)=\int_{0}^{\pi / 2}\left(-\frac{1}{2} u^{\prime 2}+u\right) d t+\int_{\pi / 2}^{\pi}\left(-\frac{1}{2} u^{\prime 2}-u\right) d t
$$

For the first approximation assume that $u(t)$ is in the following form:

$$
u(t)=A \cos \omega t
$$

Substitute this first approximation into (3.3):

$$
J(A, \omega)=2 A-\frac{1}{4} \pi A^{2} \omega^{2} .
$$

The stationary condition with respect to $A$ reads

$$
\frac{\partial J}{\partial A}=2-\frac{\pi A}{2} \omega^{2}=0,
$$

which leads to the result

$$
\omega=\frac{2}{\sqrt{A \pi}}=\frac{1.128379}{\sqrt{A}}
$$

and the approximate period can be obtained as follows:

$$
T_{\text {lapp }}(A)=\pi \sqrt{\pi A}=5.568328 \sqrt{A}, \quad \text { relative error }=1.6 \%
$$

This solution agrees with Liu's solution obtained by He's modified Lindsted-Poincaré method [16], Rafei et al.'s solution obtained by He's variational iteration method [2], Wu et al.'s solution obtained by the low-order harmonic balance method [25], and A. Belendéz et al.'s solution obtained by He's homotopy perturbation method [9].

Secondly, to obtain a more accurate result, define $u$ as follows:

$$
u=A \cos \omega t+B(\cos \omega t-\cos 3 \omega t) .
$$

Notice that (3.9) satisfies the initial conditions (3.2). 
Substituting (3.9) into (3.3), we obtain

$$
\begin{aligned}
J(A, B, \omega)= & \int_{0}^{T / 4}\left(-\frac{1}{2}(A+B)^{2} \omega^{2} \sin ^{2} \omega t+3 B(A+B) \omega^{2} \sin \omega t \sin 3 \omega t\right) d t \\
& +\int_{0}^{T / 4}\left(-\frac{9}{2} B^{2} \omega^{2} \sin ^{2} 3 \omega t+(A+B) \cos \omega t-B \cos 3 \omega t\right) d t \\
& +\int_{T / 4}^{T / 2}\left(-\frac{1}{2}(A+B)^{2} \omega^{2} \sin ^{2} \omega t+3 B(A+B) \omega^{2} \sin \omega t \sin 3 \omega t\right) d t \\
& +\int_{T / 4}^{T / 2}\left(-\frac{9}{2} B^{2} \omega^{2} \sin ^{2} 3 \omega t-(A+B) \cos \omega t+B \cos 3 \omega t\right) d t
\end{aligned}
$$

The $J(A, B, \omega)$ in (3.10) can be obtained as follows:

$$
J(A, B, \omega)=2 A+\frac{8}{3} B-\frac{1}{4} \pi A^{2} \omega^{2}-\frac{1}{2} \pi A B \omega^{2}-\frac{5}{2} \pi B^{2} \omega^{2} .
$$

The stationary condition with respect to $A$ and $B$ reads

$$
\begin{array}{ll}
\frac{\partial J}{\partial A}=2-\frac{1}{2} A \pi \omega^{2}-\frac{1}{2} B \pi \omega^{2}=0, & A=\frac{104}{27 \pi \omega^{2}} \\
\frac{\partial J}{\partial B}=\frac{8}{3}-\frac{1}{2} A \pi \omega^{2}-5 B \pi \omega^{2}=0, & B=\frac{4}{27 \pi \omega^{2}}
\end{array}
$$

from which the relationship between oscillator frequency and amplitude can be determined.

From (3.12) we have

$$
\omega=\frac{2}{3} \sqrt{\frac{26}{3 A \pi}}=\frac{1.10729}{\sqrt{A}}
$$

and the approximate period can be obtained as follows:

$$
T_{\text {2app }}(A)=\sqrt{\frac{27 A \pi^{3}}{26}}=5.67440 \sqrt{A}, \quad \text { relative error }=0.31 \%
$$

In this study, we obtained the relative error as $1.6 \%$ for the first-order approximation while the other researchers $[2,16]$ obtained the relative error as $1.8 \%$. The reason for the difference in the relative error is that the other researchers take less precision in the decimal numbers during calculations. In [9], the frequency $\omega=1.107452 / \sqrt{A}$ and the period $T_{\text {2app }}(A)=5.67440 \sqrt{A}$ were found for the same problem by second-order approximation and the relative error was calculated as $\% 0.30$.

Equation (3.1) was approximately solved in [25] using an improved harmonic balance method that incorporates salient features of both Newtons's method and the harmonic 


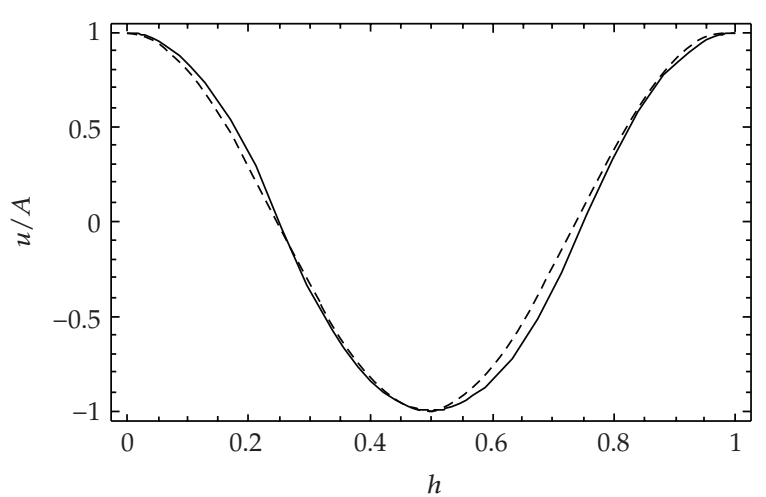

(a)

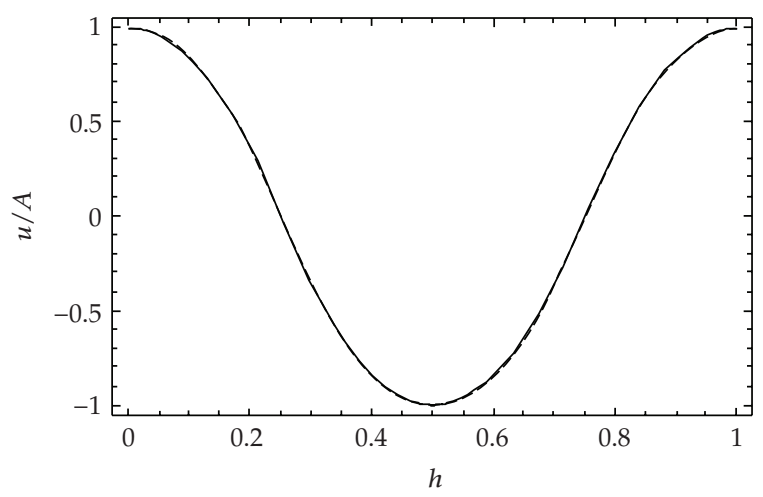

(b)

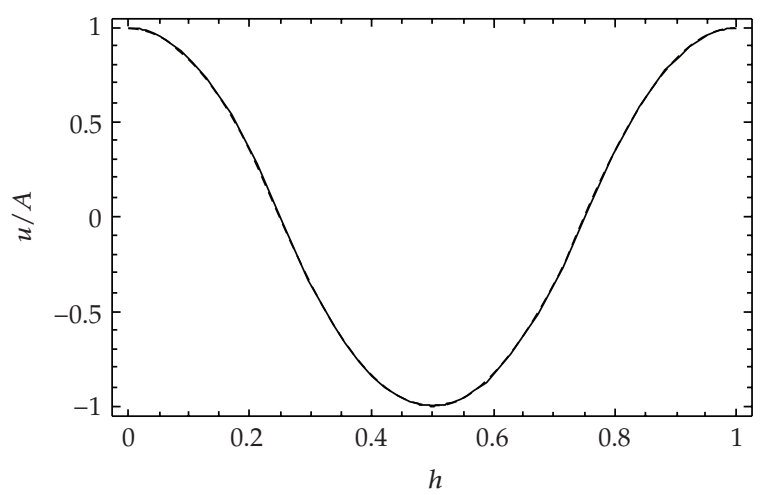

(c)

Figure 1: Comparison of approximate normalized functions (dashed line) with exact normalized functions (continuous line) for (a) first approximation (b) second approximation (c) third approximation.

balance method. In [25], the following results for the first and second-order approximations were obtained:

$$
\begin{aligned}
& T_{\text {WSL1 }}(A)=5.568328 \sqrt{A}, \quad \text { relative error }=1.6 \%, \\
& T_{\text {WSL2 }}(A)=5.67440 \sqrt{A}, \quad \text { relative error }=0.31 \% .
\end{aligned}
$$


To obtain a more accurate result, define $u$ as follows:

$$
u=A \cos \omega t+B(\cos \omega t-\cos 3 \omega t)+C(\cos 3 \omega t-\cos 5 \omega t) .
$$

Notice that (3.17) satisfies the initial conditions (3.2).

Substituting (3.17) into (3.3) gives

$J(A, B, C, \omega)=2 A+\frac{8}{3} B-\frac{16}{15} C-\frac{1}{4} \pi \omega^{2}\left(A^{2}+2 A B+10 B^{2}-18 B C+34 C^{2}\right)$.

The stationary condition with respect to $A, B$, and $C$ reads

$$
\begin{aligned}
& \frac{\partial J}{\partial A}=2-\frac{1}{2} A \pi \omega^{2}-\frac{1}{2} B \pi \omega^{2}=0, \quad A=\frac{13108}{3375 \pi \omega^{2}} \\
& \frac{\partial J}{\partial B}=\frac{8}{3}-\frac{1}{2} A \pi \omega^{2}-5 B \pi \omega^{2}+\frac{9}{2} C \pi \omega^{2}=0, \quad B=\frac{392}{3375 \pi \omega^{2}} \\
& \frac{\partial J}{\partial C}=-\frac{16}{15}-17 C \pi \omega^{2}+\frac{9}{2} B \pi \omega^{2}=0, \quad C=\frac{-4}{125 \pi \omega^{2}} .
\end{aligned}
$$

Hence the approximate frequency is

$$
\omega=\sqrt{\frac{13108}{3375 A \pi}}=\frac{1.11188}{\sqrt{A}} .
$$

Therefore, approximate period of the nonlinear oscillator can be obtained as follows:

$$
T_{3 \text { app }}(A)=2 \sqrt{\frac{3375 \pi^{3} A}{13108}}=5.65098 \sqrt{A}, \quad \text { relative error }=0.1 \%
$$

For this nonlinear problem in (3.1), the exact period is given as follows [25]:

$$
T_{e}(A)=4 \sqrt{2 A}=5.656854 \sqrt{A} .
$$

The period values and these relative errors obtained in this method for nonlinear oscillator with discontinuity are the following:

$$
\begin{aligned}
& T_{\text {1app }}(A)=5.568328 \sqrt{A}, \quad \text { relative error }=1.6 \%, \\
& T_{\text {2app }}(A)=5.67440 \sqrt{A}, \quad \text { relative error }=0.31 \%, \\
& T_{3 \text { app }}(A)=5.65098 \sqrt{A}, \quad \text { relative error }=0.10 \% .
\end{aligned}
$$


Equation (3.1) was approximately solved in [25] using an improved harmonic balance method that incorporates salient features of both Newtons's method and the harmonic balance method. In [25], the following result for the third-order approximations was obtained:

$$
T_{\text {WSL3 }}(A)=5.650976 \sqrt{A}, \quad \text { relative error }=0.10 \% \text {. }
$$

Equation (3.1) was approximately solved in [9] using a homotopy perturbation method. In [9], the following result for the third-order approximations was obtained:

$$
T_{3}(A)=5.653609 \sqrt{A}, \quad \text { relative error }=0.057 \%
$$

By using above values, the periodic function $u(t)$ can be written for three levels of approximation as follows:

$$
\begin{gathered}
u_{a 1}(t)=A \cos \left(\omega_{1} t\right), \\
u_{a 2}(t)=1.03846 A \cos \left(\omega_{2} t\right)-0.0384615 A \cos \left(3 \omega_{2} t\right), \\
u_{a 3}(t)=1.02991 A \cos \left(\omega_{3} t\right)-0.0381446 A \cos \left(3 \omega_{3} t\right)+0.00823924 A \cos \left(5 \omega_{3} t\right) .
\end{gathered}
$$

The normalized exact periodic solution $u_{\mathrm{ex}} / A$ has been obtained by numerically integrating (3.1) and (3.2) and compared with approximate solutions (3.26) in Figure 1. Here nondimensional time $h$ is defined as follows:

$$
h=\frac{t}{T_{e}} .
$$

\section{Conclusions}

He's variational approach is modified for nonlinear oscillator with discontinuities. The method has been applied to obtain three levels of approximation of a nonlinear oscillator with discontinuities for which the elastic force term is proportional to $\operatorname{sgn}(\mathrm{u})$. We reached $1.6 \%, 0.31 \%$, and $0.1 \%$ relative errors for the first, second, and third approximate periods, respectively. One can obtain higher-order accuracy by extending the idea given in this paper.

\section{References}

[1] J.-H. He and X.-H. Wu, "Variational iteration method: new development and applications," Computers E Mathematics with Applications, vol. 54, no. 7-8, pp. 881-894, 2007.

[2] M. Rafei, D. D. Ganji, H. Daniali, and H. Pashaei, "The variational iteration method for nonlinear oscillators with discontinuities," Journal of Sound and Vibration, vol. 305, no. 4-5, pp. 614-620, 2007.

[3] L.-N. Zhang and J.-H. He, "Resonance in Sirospun yarn spinning using a variational iteration method," Computers \& Mathematics with Applications, vol. 54, no. 7-8, pp. 1064-1066, 2007.

[4] T. Öziş and A. Yıldırım, "A study of nonlinear oscillators with $\mathrm{u}^{1 / 3}$ force by He's variational iteration method," Journal of Sound and Vibration, vol. 306, no. 1-2, pp. 372-376, 2007.

[5] J.-H. He, "Variational iteration method-some recent results and new interpretations," Journal of Computational and Applied Mathematics, vol. 207, no. 1, pp. 3-17, 2007. 
[6] J.-H. He, "Variational iteration method-a kind of non-linear analytical technique: some examples," International Journal of Non-Linear Mechanics, vol. 34, no. 4, pp. 699-708, 1999.

[7] H. Ozer, "Application of the variational iteration method to the boundary value problems with jump discontinuities arising in solid mechanics," International Journal of Nonlinear Sciences and Numerical Simulation, vol. 8, no. 4, pp. 513-518, 2007.

[8] A. Beléndez, C. Pascual, M. Ortuño, T. Beléndez, and S. Gallego, "Application of a modified He's homotopy perturbation method to obtain higher-order approximations to a nonlinear oscillator with discontinuities," Nonlinear Analysis: Real World Applications, vol. 10, no. 2, pp. 601-610, 2009.

[9] A. Beléndez, A. Hernandez, T. Beléndez, C. Neipp, and A. Marquez, "Higher accuracy analytical approximations to a nonlinear oscillator with discontinuity by He's homotopy perturbation method," Physics Letters A, vol. 372, no. 12, pp. 2010-2016, 2008.

[10] A. Beléndez, C. Pascual, S. Gallego, M. Ortuño, and C. Neipp, "Application of a modified He's homotopy perturbation method to obtain higher-order approximations of an $x^{1 / 3}$ force nonlinear oscillator," Physics Letters A, vol. 371, no. 5-6, pp. 421-426, 2007.

[11] A. Beléndez, C. Pascual, T. Beléndez, and A. Hernández, "Solution for an anti-symmetric quadratic nonlinear oscillator by a modified He's homotopy perturbation method," Nonlinear Analysis: Real World Applications, vol. 10, no. 1, pp. 416-427, 2009.

[12] A. Beléndez, T. Beléndez, A. Marquez, and C. Neipp, "Application of He's homotopy perturbation method to conservative truly nonlinear oscillators," Chaos, Solitons \& Fractals, vol. 37, no. 3, pp. 770780, 2008.

[13] J.-H. He, "The homotopy perturbation method nonlinear oscillators with discontinuities," Applied Mathematics and Computation, vol. 151, no. 1, pp. 287-292, 2004.

[14] A. Beléndez, A. Hernández, T. Beléndez, E. Fernández, M. L. Álvarez, and C. Neipp, "Application of He's homotopy perturbation method to the duffin-harmonic oscillator," International Journal of Nonlinear Sciences and Numerical Simulation, vol. 8, no. 1, pp. 79-88, 2007.

[15] T. Özis and A. Yildırım, "A comparative study of He's homotopy perturbation method for determining frequency-amplitude relation of a nonlinear oscillator with discontinuities," International Journal of Nonlinear Sciences and Numerical Simulation, vol. 8, no. 2, pp. 243-248, 2007.

[16] H.-M. Liu, "Approximate period of nonlinear oscillators with discontinuities by modified LindstedtPoincare method," Chaos, Solitons \& Fractals, vol. 23, no. 2, pp. 577-579, 2005.

[17] S.-Q. Wang and J.-H. He, "Nonlinear oscillator with discontinuity by parameter-expansion method," Chaos, Solitons \& Fractals, vol. 35, no. 4, pp. 688-691, 2008.

[18] F. Ö. Zengin, M. O. Kaya, and S. A. Demirbağ, "Application of parameter-expansion method to nonlinear oscillators with discontinuities," International Journal of Nonlinear Sciences and Numerical Simulation, vol. 9, no. 3, pp. 267-270, 2008.

[19] S. Momani and S. Abuasad, "Application of He's variational iteration method to Helmholtz equation," Chaos, Solitons \& Fractals, vol. 27, no. 5, pp. 1119-1123, 2006.

[20] J.-H. He, "Variational approach for nonlinear oscillators," Chaos, Solitons \& Fractals, vol. 34, no. 5, pp. 1430-1439, 2007.

[21] J.-H. He, "Variational principles for some nonlinear partial differential equations with variable coefficients," Chaos, Solitons \& Fractals, vol. 19, no. 4, pp. 847-851, 2004.

[22] J.-H. He, "An elementary introduction to recently developed asymptotic methods and nanomechanics in textile engineering," International Journal of Modern Physics B, vol. 22, no. 21, pp. 3487-3578, 2008.

[23] D.-H. Shou, "Variational approach to the nonlinear oscillator of a mass attached to a stretched wire," Physica Scripta, vol. 77, no. 4, Article ID 045006, 4 pages, 2008.

[24] Z.-L. Tao, "The frequency-amplitude relationship for some nonlinear oscillators with discontinuity by He's variational method," Physica Scripta for Experimental and Theoretical Physics, vol. 78, no. 1, Article ID 015004, 2 pages, 2008.

[25] B. S. Wu, W. P. Sun, and C. W. Lim, "An analytical approximate technique for a class of strongly non-linear oscillators," International Journal of Non-Linear Mechanics, vol. 41, no. 6-7, pp. 766-774, 2006. 


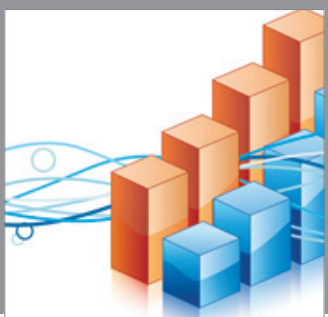

Advances in

Operations Research

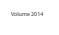

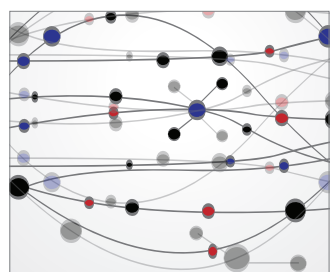

\section{The Scientific} World Journal
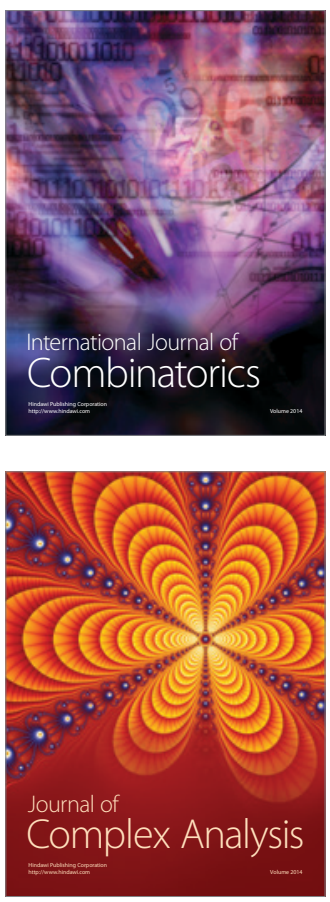

International Journal of

Mathematics and

Mathematical

Sciences
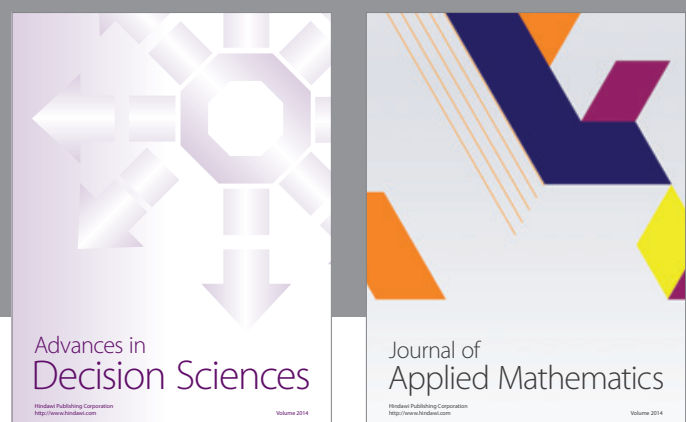

Journal of

Applied Mathematics
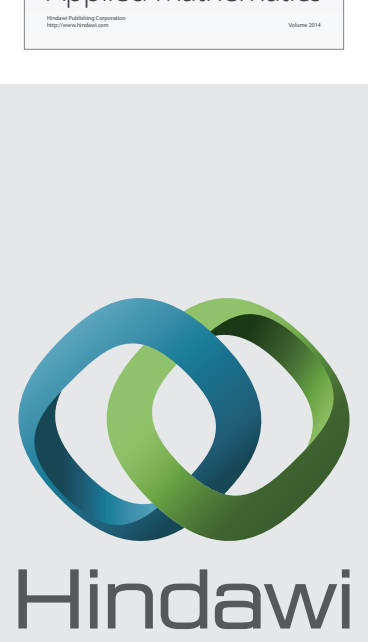

Submit your manuscripts at http://www.hindawi.com
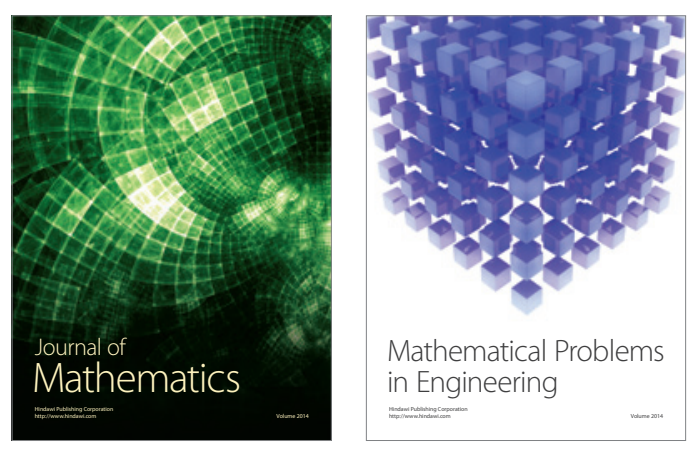

Mathematical Problems in Engineering
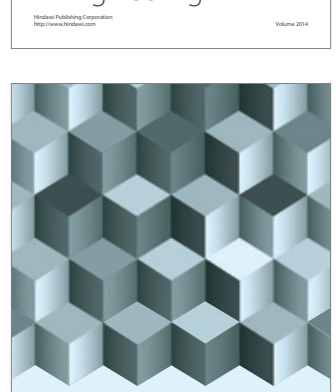

Journal of

Function Spaces
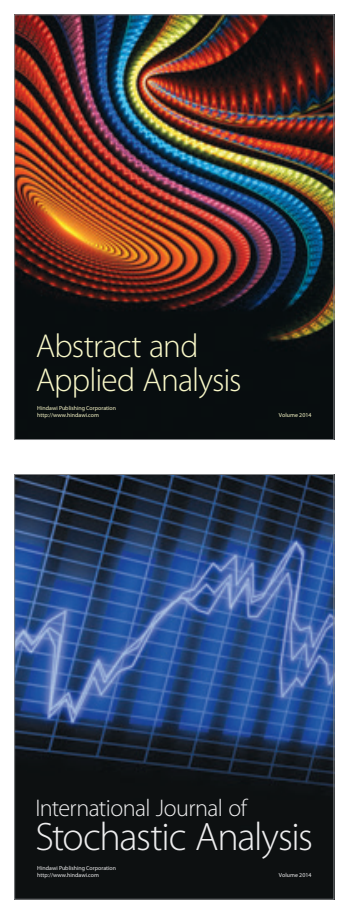

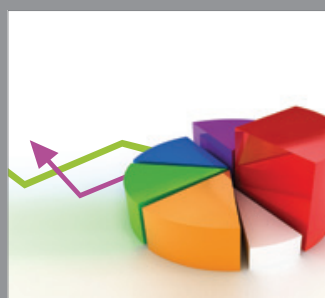

ournal of

Probability and Statistics

Promensencen
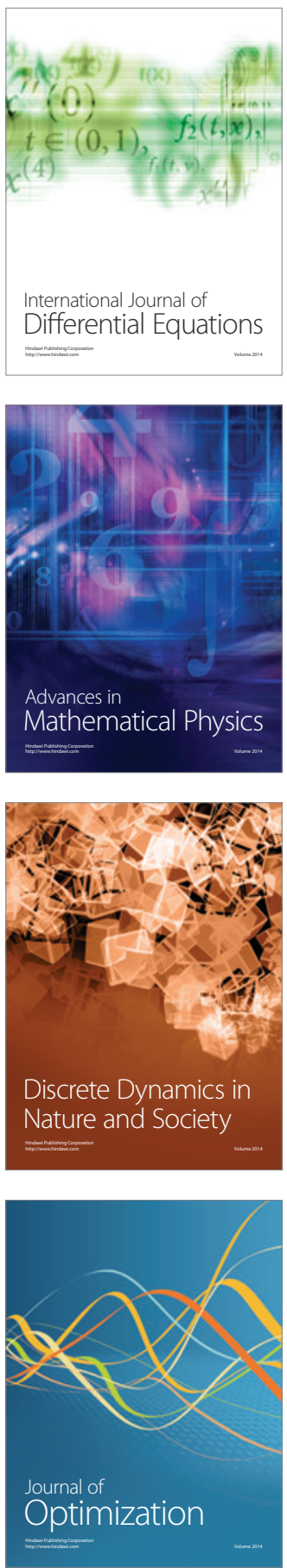